\title{
Fractals and Chaos Related to Ising-Onsager- Zhang Lattices. Quaternary Approach vs. Ternary Approach
}

\author{
J. Ławrynowicz, O. Suzuki, A. Niemczynowicz* (1) and \\ M. Nowak-Kȩpczyk
}

In memory of Professor W. A. Rodrigues Jr.

\begin{abstract}
Continuing our idea (Int. J. Geom. Methods Mod. Phys.) of extending some aspects of Onsager (Phys Rev 65:117-149, 1944) crystal statistics to three dimensions taking into account binary and ternary crystal structures in connection with fractals and chaos related to IsingOnsager-Zhang lattices, we (1) use the Galois extension structure of the nonion algebra, (2) analyze ternary and binary structures of $\mathfrak{s u}(3)$ as well as (3) analyze the identification of the construction of the collection of two ternaries with the collection of three binaries, (4) observe that the approach is applicable to quarks and elementary particles including introduction of colors and, finally, (5) suggest an analysis of three quaternaries vs. four ternaries, involving duodevicenion and/or quindenion algebra.
\end{abstract}

Mathematics Subject Classification. Primary 82C44; Secondary 82D25, 81R05, 15A66.

Keywords. Crystal lattice, Ising lattice, Octonions, Quaternions, Sedenions, Nonions, Binary structures, Ternary alloy, Composition algebra.

\section{Introduction and Motivation}

In order to develop further our previous idea [1]; Eawrynowicz et al. [16] of identification of two ternaries and three binaries with the help of noncommutative Galois extension applied to fractals and chaos related to Ising

This article is part of the Topical Collection on Homage to Prof. W.A. Rodrigues Jr. edited by Jayme Vaz Jr..

${ }^{*}$ Corresponding author. 
[2]-Onsager [3]-Zhang [4] lattices, in this considerations we start with involving the Galois extension structure of the nonion algebra and analyzing the ternary and binary structures of $\mathfrak{s u}(3)$. Our approach is also motivated by unclear statements in the Zhang [4] paper and further treatise [5,6]. The main results of this paper are summarized as Theorems $1-3$. Next we analyze the identification of construction of the collection of two ternaries with the collection of three binaries, and observe that the approach is applicable to quarks and elementary particles including introduction of colours. Our results announced above provide an example of a mathematical procedure applicable parallelly in the physics of condensed matter and physics of elementary particles. In this place it seems naturally to quote the suprising results due to [58-65] dealing with discovery of topological phase transition and topological phases matter [57].

Finally we suggest an analysis of three quaternaries vs. four ternaries, involving duodevicenion and/or quindenion algebra. More precisely, we suggest the construction of the collection of four quaternaries and the corresponding Dirac-like operators in connection with the nonion algebra. We suggest to construct then the collection of four quaternaries and the corresponding Dirac-like operators in connection with the duodevicenion or quindenion algebra [52-54] and its ternary extension. The next steps suggested are noncommutative Galois extensions and a study of their basic relations and Galois extensions of ternary Clifford type [67], quaternary Galois extensions and Galois extensions of quaternary Clifford type [9], the Galois extension structure of the duodevicenion and quindenion algebras, quaternary and ternary structure of $\mathfrak{s u}(3)$, ternary and quaternary Dirac-like operators of noncommutative Galois extensions [26,27,68] and, finally, identification of the constructed collection of three quaternaries with the proper collection of four ternaries.

An approach of ternary numbers, algebras, and complex analysis, coming back to geometric ideas suggested in [66].

\section{The Galois Extension Structure of the Nonion Algebra}

We recall the concept of nonion algebra $\mathbb{N}[52-54]$ and discuss its Galois extension structure.

Consider the following matrices

$$
\begin{aligned}
Q_{1}=\left(\begin{array}{lll}
0 & \mathbf{j} & 0 \\
0 & 0 & \mathbf{j}^{2} \\
1 & 0 & 0
\end{array}\right), Q_{2}=\left(\begin{array}{lll}
0 & \mathbf{j}^{2} & 0 \\
0 & 0 & \mathbf{j} \\
1 & 0 & 0
\end{array}\right), Q_{3}=\left(\begin{array}{lll}
0 & 1 & 0 \\
0 & 0 & 1 \\
1 & 0 & 0
\end{array}\right) ; \\
\bar{Q}_{1}=\left(\begin{array}{lll}
0 & 0 & 1 \\
\mathbf{j}^{2} & 0 & 0 \\
0 & \mathbf{j} & 0
\end{array}\right), \bar{Q}_{2}=\left(\begin{array}{ccc}
0 & 0 & 1 \\
\mathbf{j} & 0 & 0 \\
0 & \mathbf{j}^{2} & 0
\end{array}\right), \bar{Q}_{3}=\left(\begin{array}{lll}
0 & 0 & 1 \\
1 & 0 & 0 \\
0 & 1 & 0
\end{array}\right) ; \\
R_{1}=\left(\begin{array}{lll}
1 & 0 & 0 \\
0 & 1 & 0 \\
0 & 0 & 1
\end{array}\right), R_{2}=\left(\begin{array}{ccc}
1 & 0 & 0 \\
0 & \mathbf{j} & 0 \\
0 & 0 & \mathbf{j}^{2}
\end{array}\right), R_{3}=\left(\begin{array}{ccc}
1 & 0 & 0 \\
0 & \mathbf{j}^{2} & 0 \\
0 & 0 & \mathbf{j}
\end{array}\right) .
\end{aligned}
$$


where $\mathbf{j}$ is one of the roots of $z^{3}-1=0$ different from 1 . The matrix algebra which is generated by two of the three elements (2.1) over $\mathbb{R}\left[\sqrt[3]{I_{3}}\right]$ is called nonion algebra $\mathbb{N}$.

Consider in addition the matrices of the form

$$
\begin{aligned}
& T_{1}=I_{6}:=\left(\begin{array}{ll}
I_{3} & 0_{3} \\
0_{3} & I_{3}
\end{array}\right), \quad T_{2}=\left(\begin{array}{cc}
T_{1}^{0} & 0_{3} \\
0_{3} & T_{1}^{0}
\end{array}\right), T_{3}=\left(\begin{array}{cc}
T_{2}^{0} & 0_{3} \\
0_{3} & T_{2}^{0}
\end{array}\right), \\
& T_{4}=\left(\begin{array}{cc}
0_{3} & I_{3} \\
-I_{3} & 0_{3}
\end{array}\right), \quad T_{5}=\left(\begin{array}{cc}
0_{3} & T_{1}^{0} \\
-T_{1}^{0} & 0_{3}
\end{array}\right), \quad T_{6}=\left(\begin{array}{cc}
0_{3} & T_{2}^{0} \\
-T_{2}^{0} & 0_{3}
\end{array}\right),
\end{aligned}
$$

where

$$
T_{1}^{0}=\left(\begin{array}{lll}
0 & 1 & 0 \\
0 & 0 & 1 \\
1 & 0 & 0
\end{array}\right), \quad T_{2}^{0}=\left(\begin{array}{lll}
0 & 0 & 1 \\
1 & 0 & 0 \\
0 & 1 & 0
\end{array}\right) .
$$

We recall that the nine elements (2.1)-(2.3) constitute linear basis of the nonion algebra $\mathbb{N}$. The matrix algebra which is generated by two of the three elements (2.5) over the real field $\mathbb{R}$ will be denoted by $\mathbb{B}^{\prime}$. The algebra generated by $T_{1}^{0}$ or $T_{2}^{0}$ is called cubic algebra, denoted by $\mathbb{B}$. The algebra $\widetilde{\mathbb{N}}$ generated by $T_{4}$ is the binary extension of $\mathbb{N}$,

$$
\widetilde{\mathbb{N}}=\mathbb{N}\left[\sqrt[2]{I_{3}}\right]: \widetilde{\mathbb{N}}=\left\{x+y T_{4} \mid x, y \in \mathbb{N}\right\} .
$$

The three elements (2.4) are a linear basis of $\mathbb{B}$. The six elements (2.4), (2.5) form a linear basis of $\mathbb{B}^{\prime}$. Besides, $\mathbb{B}$ and $\mathbb{B}^{\prime}$ are subalgebras of $\mathbb{N}$ and $\widetilde{\mathbb{N}}$ respectively. We can prove the above assertions with the help of the two enclosed product tables.

Now, owing to hints [13] and motivation given in references [7, 8,13,15], following some earlier demands appearing in references $[11,12,16-18]$, we can prove the following basic theorems on the Galois extensions.

Theorem 1. (1) The nonion algebra is a ternary Galois extension of the algebra $\mathbb{B}: \mathbb{N}=\mathbb{B}\left[\sqrt[3]{I_{3}}\right]$. The extension can be realized by $\mathbb{B}[\tau]\left(\tau^{3}=I_{3}\right)$ with the choice of $\tau=Q_{i}, \bar{Q}_{i}(i=1,2,3)$. (2) $\widetilde{\mathbb{N}}$ is a binary extension of $\mathbb{B}^{\prime}: \widetilde{\mathbb{N}}=\mathbb{B}^{\prime}\left[\sqrt[2]{I_{3}}\right]$. Hence we have the following commutative diagram:

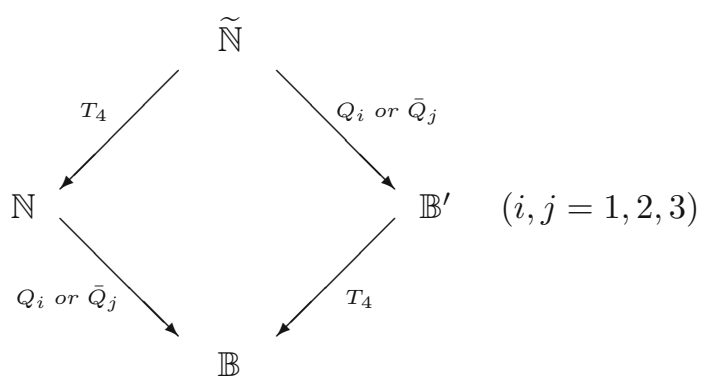

Proof will be given in the next section. 
Theorem 2. (3) We have the following Galois extensions:

$$
\left\{\begin{array}{l}
\mathbb{A}\left[R_{1}, R_{2}, R_{3}\right]=\left\{x R_{1}+y R_{2}+z R_{3} \mid x, y, z \in \mathbb{R}[\mathbf{j}]\right\}, \\
\mathbb{A}\left[R_{1}, Q_{i}, \bar{Q}_{i}\right]=\left\{x R_{1}+y Q_{i}+z \bar{Q}_{i} \mid x, y, z \in \mathbb{R}[\mathbf{j}]\right\}, \quad(i=1,2,3), \\
\mathbb{A}\left[R_{1}, \bar{Q}_{i}, Q_{i}\right]=\left\{x R_{1}+y \bar{Q}_{i}+z Q_{i} \mid x, y, z \in \mathbb{R}[\mathbf{j}]\right\}, \quad(i=1,2,3) .
\end{array}\right.
$$

The extension does not depend on the choice of $\tau$ with $\mathbb{B}[\tau] \quad\left(\tau^{3}=I_{3}\right)$ : we have

$$
\mathbb{N}=\mathbb{A}\left[R_{1}, Q_{1}, \overline{Q_{1}}\right]=\mathbb{A}\left[R_{1}, Q_{2}, \overline{Q_{2}}\right]=\mathbb{A}\left[R_{1}, Q_{3}, \overline{Q_{3}}\right] .
$$

(4) $Q_{i}, \bar{Q}_{j}(i, j=1,2,3)$ give a part of generators of the Galois group of $\mathbb{N}: \mathbb{N}=\mathbb{B}\left[\sqrt[3]{I_{3}}\right]$. Namely putting

$$
\begin{gathered}
\mathbb{A}_{U}\left[R_{1}, R_{2}, R_{3}\right]=\left\{x R_{1}+y U R_{2}+z \bar{U} R_{3} \mid x, y, z \in \mathbb{R}[\mathbf{j}]\right\}, \\
\text { where } U=Q_{i}, \bar{Q}_{i},(i=1,2,3),
\end{gathered}
$$

we can obtain new Galois extensions

$$
\begin{cases}\mathbb{A}_{Q_{1}}\left[R_{1}, R_{2}, R_{3}\right]=\mathbb{A}\left[R_{1}, Q_{2}, \overline{Q_{2}}\right], & \mathbb{A}_{Q_{2}}\left[R_{1}, R_{2}, R_{3}\right]=\mathbb{A}\left[R_{1}, Q_{3}, \overline{Q_{3}}\right], \\ \mathbb{A}_{\bar{Q}_{1}}\left[R_{1}, R_{2}, R_{3}\right]=\mathbb{A}\left[R_{1}, Q_{3}, \overline{Q_{3}}\right], & \mathbb{A}_{\bar{Q}_{2}}\left[R_{1}, R_{2}, R_{3}\right]=\mathbb{A}\left[R_{1}, Q_{1}, \overline{Q_{1}}\right], \\ \mathbb{A}_{R_{2}}\left[R_{1}, Q_{1}, \overline{Q_{1}}\right]=\mathbb{A}\left[R_{1}, Q_{2}, \overline{Q_{2}}\right], & \mathbb{A}_{R_{2}}\left[R_{1}, Q_{2}, \overline{Q_{2}}\right]=\mathbb{A}\left[R_{1}, Q_{3}, \overline{Q_{3}}\right], \\ \mathbb{A}_{R_{2}}\left[R_{1}, Q_{3}, \overline{Q_{3}}\right]=\mathbb{A}\left[R_{1}, Q_{1}, \overline{Q_{1}}\right], & \mathbb{A}_{\bar{Q}_{1}}\left[R_{1}, R_{2}, R_{3}\right]=\mathbb{A}\left[R_{1}, Q_{3}, \overline{Q_{3}}\right], \\ \mathbb{A}_{\bar{Q}_{2}}\left[R_{1}, R_{2}, R_{3}\right]=\mathbb{A}\left[R_{1}, Q_{1}, \overline{Q_{1}}\right], & \mathbb{A}_{\bar{Q}_{3}}\left[R_{1}, R_{2}, R_{3}\right]=\mathbb{A}\left[R_{1}, Q_{2}, \overline{Q_{2}}\right] .\end{cases}
$$

(5) We have the following results for the adjoint operations:

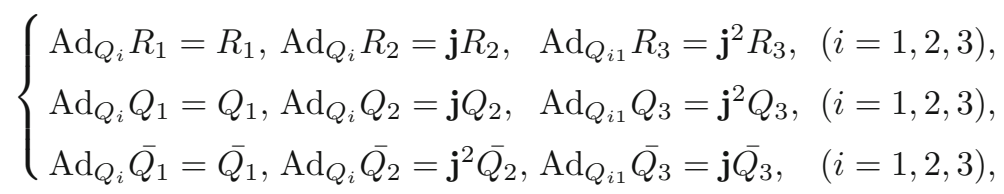

where $\mathbf{j} \neq 1, \mathbf{j}^{3}=1$.

The proof will be given in the next section.

\section{Proofs of Basic Theorems 1 and 2 [Assertions (1)-(5)]}

Proof. Ad (1). We notice that $\mathbb{B}$ is the commutative Galois extension: $\mathbb{B}=$ $\mathbb{R}\left[\sqrt[3]{I_{3}}\right]$.

Then we observe that, for a Clifford algebra $\mathcal{A}$ with generators $T_{1}, T_{2}, \ldots$, $T_{n}$, there is a sequence of noncommutative binary Galois extensions of $\mathbb{R}$ which realizes the given Clifford algebra $\mathcal{A}[19]$. 

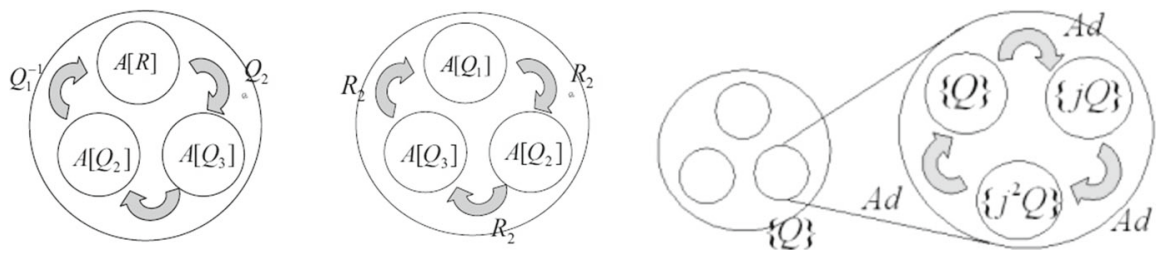

Figure 1. Schemes illustrating reasoning leading to Assertions (1)-(5)

Indeed, let us prove the above statement by induction with respect to $m$, where $\mathcal{A}_{0}=\mathbb{R}, \mathcal{A}_{m}=\mathcal{A}$, and

$$
T_{i} T_{j}+T_{j} T_{i}=-\delta_{i j} I_{n} \Rightarrow \mathcal{A}_{k}=\mathcal{A}_{k-1}\left[\sqrt[2]{-I_{n}}\right], \quad k=1,2, \ldots, m .
$$

Complex numbers can be obtained by commutative extensions of real numbers. Now, setting

$$
\hat{T}_{i}=\left(\begin{array}{cc}
T_{i} & 0 \\
0 & -T_{i}
\end{array}\right), \quad i=1,2, \ldots, m ; \quad \hat{H}_{n+1}=\left(\begin{array}{cc}
0 & I_{n} \\
-I_{n} & 0
\end{array}\right),
$$

we get Clifford algebra which is generated by $\left(\hat{T}_{1}, \hat{T}_{2}, \ldots, \hat{T}_{n}, \hat{H}_{n+1}\right)$ on one hand, and the right (or left) module binary extension of $\mathcal{A}_{n}$ by $\hat{H}_{n+1}$ on the other hand.

If we choose in (1) $\tau=Q_{i} \bar{Q}_{i}, i=1,2,3$, we make the Galois extension $\mathbb{B}\left[\sqrt[3]{I_{3}}\right]$. Then we can see that it is identical with $\mathbb{N}$.

Ad (2). We notice that $\mathbb{B}^{\prime}$ is the noncommutative Galois extension of $\mathbb{B}: \mathbb{B}^{\prime}=\mathbb{B}\left[\sqrt[2]{I_{3}}\right]$, where $\sqrt[2]{I_{3}}=T_{4}$. Choosing $\tau=T_{4}$, we make the Galois extension. Then we can see that it is identical with $\widetilde{\mathbb{N}}: \widetilde{\mathbb{N}}=\mathbb{B}^{\prime}\left[\sqrt[2]{I_{3}}\right]$.

Ad (3)-(5). Clearly, for $i=1$ we have

$$
\mathbb{R}\left[\mathbf{j}, Q_{1}\right]=\left\{x R_{1}+y Q_{1}+z Q_{1}^{2} \mid x, y, z \in \mathbb{R}[\mathbf{j}]\right\} .
$$

From $Q_{1}^{2}=\bar{Q}_{1}$ it follows that

$$
\mathbb{R}\left[\mathbf{j}, Q_{1}\right]=\left\{x R_{1}+y Q_{1}+z \bar{Q}_{1} \mid x, y, z \in \mathbb{R}[\mathbf{j}]\right\} .
$$

Hence $\mathbb{R}\left[\mathbf{j}, Q_{1}\right]$ is a ternary Galois extension with the corresponding Galois group $1, Q_{1}, Q_{1}^{2}$. Analogously we perform the reasoning for $\mathbb{R}\left[\mathbf{j}, Q_{2}\right]$ and $\mathbb{R}[\mathbf{j}$, $\left.Q_{3}\right]$.

In general, we follow the enclosed schemes (Fig. 1) which indicate how to use in the clockwise way Tables 1 and 2 . The scheme is clarified by a detailed calculation in the case expressed in the second line of the formulae (2.10).

\section{Ternary and Binary Galois Extension Structures of $\mathfrak{s u}(3)$}

In addition to the desires followed from our programme formulated in [1], the present section meets also the demands appearing in references [20-25]. We 
TABLE 1. The (Q, $\bar{Q}, \mathrm{R})$-matrices product table

\begin{tabular}{llllllllll}
\hline & $Q_{1}$ & $Q_{2}$ & $Q_{3}$ & $\bar{Q}_{1}$ & $\bar{Q}_{2}$ & $\bar{Q}_{3}$ & $R_{1}$ & $R_{2}$ & $R_{3}$ \\
\hline$Q_{1}$ & $\bar{Q}_{1}$ & $\mathbf{j}^{2} \bar{Q}_{3}$ & $\mathbf{j} \bar{Q}_{2}$ & $R_{1}$ & $\mathbf{j}^{2} R_{3}$ & $\mathbf{j} R_{2}$ & $Q_{1}$ & $Q_{2}$ & $Q_{3}$ \\
$Q_{2}$ & $\mathbf{j} \bar{Q}_{3}$ & $\bar{Q}_{2}$ & $\mathbf{j}^{2} \bar{Q}_{1}$ & $\mathbf{j} R_{2}$ & $R_{1}$ & $\mathbf{j}^{2} R_{3}$ & $Q_{2}$ & $Q_{3}$ & $Q_{1}$ \\
$Q_{3}$ & $\mathbf{j}^{2} \bar{Q}_{2}$ & $\mathbf{j} \bar{Q}_{1}$ & $\bar{Q}_{3}$ & $\mathbf{j}^{2} R_{3}$ & $\mathbf{j} R_{2}$ & $R_{1}$ & $Q_{3}$ & $Q_{1}$ & $Q_{2}$ \\
$\bar{Q}_{1}$ & $R_{1}$ & $R_{2}$ & $R_{3}$ & $Q_{1}$ & $\mathbf{j}^{2} Q_{3}$ & $\mathbf{j} Q_{2}$ & $\bar{Q}_{1}$ & $\mathbf{j}^{2} \bar{Q}_{3}$ & $\mathbf{j} \bar{Q}_{2}$ \\
$\bar{Q}_{2}$ & $R_{3}$ & $R_{1}$ & $R_{2}$ & $\mathbf{j} Q_{3}$ & $Q_{2}$ & $\mathbf{j}^{2} Q_{1}$ & $\bar{Q}_{2}$ & $\mathbf{j}^{2} \bar{Q}_{1}$ & $\mathbf{j} \bar{Q}_{3}$ \\
$\bar{Q}_{3}$ & $R_{2}$ & $R_{3}$ & $R_{1}$ & $\mathbf{j}^{2} Q_{2}$ & $\mathbf{j} Q_{1}$ & $Q_{3}$ & $\bar{Q}_{3}$ & $\mathbf{j}^{2} \bar{Q}_{2}$ & $\mathbf{j} \bar{Q}_{1}$ \\
$R_{1}$ & $Q_{1}$ & $Q_{2}$ & $Q_{3}$ & $\bar{Q}_{1}$ & $\bar{Q}_{2}$ & $\bar{Q}_{3}$ & $R_{1}$ & $R_{2}$ & $R_{3}$ \\
$R_{2}$ & $\mathbf{j}^{2} Q_{2}$ & $\mathbf{j}^{2} Q_{3}$ & $\mathbf{j}^{2} Q_{1}$ & $\bar{Q}_{3}$ & $\bar{Q}_{1}$ & $\bar{Q}_{2}$ & $R_{2}$ & $R_{3}$ & $R_{1}$ \\
$R_{3}$ & $\mathbf{j} Q_{3}$ & $\mathbf{j} Q_{1}$ & $\mathbf{j} Q_{2}$ & $\bar{Q}_{2}$ & $\bar{Q}_{3}$ & $\bar{Q}_{1}$ & $R_{3}$ & $R_{1}$ & $R_{2}$ \\
\hline
\end{tabular}

TABle 2. The T-matrices product table

\begin{tabular}{ccccccc}
\hline & $T_{1}$ & $T_{2}$ & $T_{3}$ & $T_{4}$ & $T_{5}$ & $T_{6}$ \\
\hline$T_{1}$ & $T_{1}$ & $T_{2}$ & $T_{3}$ & $T_{4}$ & $T_{5}$ & $T_{6}$ \\
$T_{2}$ & $T_{2}$ & $T_{3}$ & $T_{1}$ & $T_{5}$ & $T_{6}$ & $T_{4}$ \\
$T_{3}$ & $T_{3}$ & $T_{1}$ & $T_{2}$ & $T_{6}$ & $T_{4}$ & $T_{5}$ \\
$T_{4}$ & $T_{4}$ & $T_{6}$ & $T_{5}$ & $T_{1}$ & $T_{3}$ & $T_{2}$ \\
$T_{5}$ & $T_{5}$ & $T_{4}$ & $T_{6}$ & $T_{2}$ & $T_{1}$ & $T_{3}$ \\
$T_{6}$ & $T_{6}$ & $T_{5}$ & $T_{4}$ & $T_{3}$ & $T_{2}$ & $T_{1}$ \\
\hline
\end{tabular}

are going to discuss the structure of the Galois extension in the context of $\mathfrak{s u}(3)$.

We recall base of $\mathfrak{s u}(3)$ (where selected generators are proportional to the well known Gell-Mann matrices [14]):

$$
\begin{aligned}
f_{1} & =\left(\begin{array}{lll}
0 & i & 0 \\
i & 0 & 0 \\
0 & 0 & 0
\end{array}\right), f_{2}=\left(\begin{array}{ccc}
0 & -1 & 0 \\
1 & 0 & 0 \\
0 & 0 & 0
\end{array}\right), f_{3}=\left(\begin{array}{ccc}
i & 0 & 0 \\
0 & -i & 0 \\
0 & 0 & 0
\end{array}\right), \\
f_{4} & =\left(\begin{array}{lll}
0 & 0 & i \\
0 & 0 & 0 \\
i & 0 & 0
\end{array}\right), \\
f_{5} & =\left(\begin{array}{ccc}
0 & 0 & -1 \\
0 & 0 & 0 \\
1 & 0 & 0
\end{array}\right), f_{6}=\left(\begin{array}{ccc}
0 & 0 & 0 \\
0 & 0 & i \\
0 & i & 0
\end{array}\right), f_{7}=\left(\begin{array}{ccc}
0 & 0 & 0 \\
0 & 0 & -1 \\
0 & 1 & 0
\end{array}\right) \\
f_{8} & =\frac{1}{\sqrt{3}}\left(\begin{array}{ccc}
1 & 0 & 0 \\
0 & 1 & 0 \\
0 & 0 & -2
\end{array}\right)
\end{aligned}
$$


and construct the three linear subspaces:

$$
\left\{\begin{array}{l}
L_{1}: \quad e_{1}=\left(\begin{array}{lll}
0 & i & 0 \\
i & 0 & 0 \\
0 & 0 & 0
\end{array}\right), e_{2}=\left(\begin{array}{ccc}
0 & -1 & 0 \\
1 & 0 & 0 \\
0 & 0 & 0
\end{array}\right), e_{3}=\left(\begin{array}{ccc}
i & 0 & 0 \\
0 & -i & 0 \\
0 & 0 & 0
\end{array}\right), \\
L_{2}: e_{1}^{\prime}=\left(\begin{array}{lll}
0 & 0 & i \\
0 & 0 & 0 \\
i & 0 & 0
\end{array}\right), e_{2}^{\prime}=\left(\begin{array}{ccc}
0 & 0 & 1 \\
0 & 0 & 0 \\
-1 & 0 & 0
\end{array}\right), e_{3}^{\prime}=\left(\begin{array}{ccc}
-i & 0 & 0 \\
0 & 0 & 0 \\
0 & 0 & i
\end{array}\right), \\
L_{3}: \quad e_{1}^{\prime \prime}=\left(\begin{array}{lll}
0 & 0 & 0 \\
0 & 0 & i \\
0 & i & 0
\end{array}\right), e_{2}^{\prime \prime}=\left(\begin{array}{ccc}
0 & 0 & 0 \\
0 & 0 & -1 \\
0 & 1 & 0
\end{array}\right), e_{3}^{\prime \prime}=\left(\begin{array}{ccc}
0 & 0 & 0 \\
0 & -i & 0 \\
0 & 0 & i
\end{array}\right) .
\end{array}\right.
$$

We notice the relation

$$
f_{8}=\frac{-1}{i \sqrt{3}}\left(e_{3}^{\prime}+e_{3}^{\prime \prime}\right)
$$

while $\left\{e_{3}, e_{3}^{\prime}, e_{3}^{\prime \prime}\right\}$ is linearly dependent. Hence we can see that $\left(e_{1}, e_{2}, \ldots, e_{3}^{\prime \prime}\right)$ constitute the basis omitting one of $e_{3}, e_{3}^{\prime}, e_{3}^{\prime \prime}$.

We can prove the following basic theorem on both binary and ternary Galois extension structures on $\mathfrak{s u}(3)$, extremely important from the point of view of the ${ }_{4}$ of our research programme formulated in Section 4 of [69]: identification of two ternaries with three binaries with the help of noncommutative Galois extensions; cf. [26,27]:

Theorem 3. We have the binary and ternary extension structures on $\mathfrak{s u}(3)$ :

(6) We have the following adjoint representation on $L_{i}(i=1,2,3)$ :

$$
\left\{\begin{array}{lll}
H e_{1} H^{-1}=-e_{2}, & H e_{2} H^{-1}=e_{1}, & H e_{3} H^{-1}=e_{3}, \\
H^{\prime} e_{1}^{\prime} H^{\prime-1}=-e_{2}^{\prime}, & H^{\prime} e_{2}^{\prime} H^{\prime-1}=-e_{1}^{\prime}, & H^{\prime} e_{3}^{\prime} H^{\prime-1}=e_{3}^{\prime}, \\
H^{\prime} e_{1}^{\prime \prime} H^{\prime-1}=e_{2}^{\prime \prime}, & H^{\prime} e_{2}^{\prime \prime} H^{\prime-1}=e_{1}^{\prime \prime}, & H^{\prime} e_{3}^{\prime \prime} H^{\prime-1}=e_{3}^{\prime \prime},
\end{array}\right.
$$

where

$$
H=\left(\begin{array}{lll}
1 & 0 & 0 \\
0 & i & 0 \\
0 & 0 & 1
\end{array}\right), \quad H^{\prime}=\left(\begin{array}{lll}
1 & 0 & 0 \\
0 & 1 & 0 \\
0 & 0 & i
\end{array}\right)
$$

(7) We can obtain the following commutation relations:

$$
\left\{\begin{array}{l}
e_{1}^{2}=e_{2}^{2}=e_{3}^{2}=-1 \\
e_{1} e_{2}=-e_{2} e_{1}=e_{3}, e_{2} e_{3}=-e_{3} e_{2}=e_{1}, e_{3} e_{1}=-e_{1} e_{3}=e_{2} .
\end{array}\right.
$$

After the central extension, we have the Clifford algebra which is isomorphic to the quaternion algebra. For the case of $e_{i}^{\prime}$ and $e_{i}^{\prime \prime}(i=1,2,3)$, we have the same assertions on $L_{i}(i=1,2,3)$. Hence we obtain the Dirac-like operators desired.

(8) We have

$$
\begin{aligned}
& G_{1} e_{k} G_{1}^{-1}=e_{k}^{\prime}(k=1,2,3), G_{1} e_{k}^{\prime} G_{1}^{-1}=e_{k}^{\prime \prime}(k=1,2), G_{1} e_{3}^{\prime} G_{1}^{-1}=-e_{3}^{\prime \prime} \\
& G_{1} e_{k}^{\prime \prime} G_{1}^{-1}=e_{k}(k=1,3), G_{1} e_{2}^{\prime \prime} G_{1}^{-1}=-e_{2},
\end{aligned}
$$



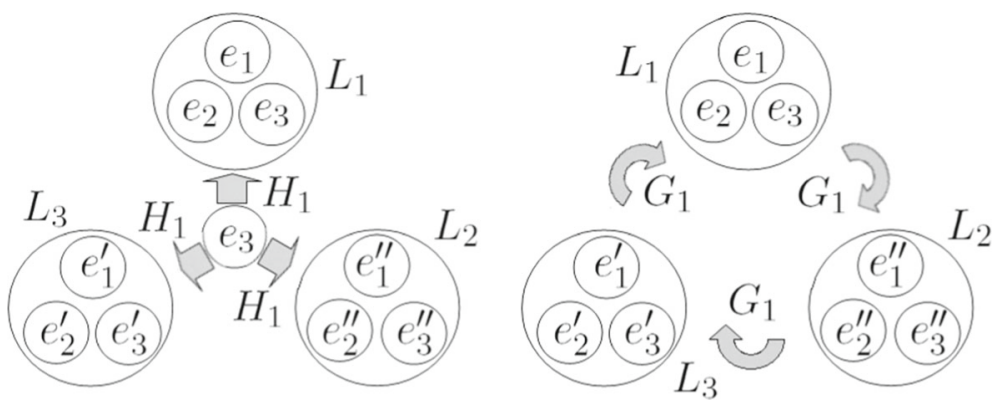

Figure 2. Schematic comparison of the commutative

Galois extension structures on $\mathfrak{s u}(3)$

where

$$
G_{1}=\left(\begin{array}{lll}
0 & 1 & 0 \\
0 & 0 & 1 \\
1 & 0 & 0
\end{array}\right)
$$

By this result, we can find a ternary commutative Galois extension. Hence we can introduce the ternary Dirac-like operators.

Proof. Basic elements of our considerations are summarized as properties (1) of $s u(3),(2)$ of $\mathrm{su}(2)$, and (3) of $\mathfrak{s u}(3)$; as schematic comparison of the commutative Galois extension structures on $\mathfrak{s u}(3)$ (Fig. 2), and as the diagram (4.7):

(1) $s u(3)=L_{1} \cup L_{2} \cup L_{3}$ $s u(3)$

(2) $L_{i}(i=1,2,3)$ is isomorphic to $\mathrm{su}(2)$ and it is a binary $\mathrm{su}(2)$ Galois extension $L_{i}=B_{0}\left[\sqrt[2]{I_{3}}\right]$ over $B_{0}=R\left[e_{3}\right]$ $\downarrow \sqrt[2]{I_{3}}$

(3) $\mathfrak{s u}(3)$ is a ternary Galois extension $B^{\prime}\left[\sqrt[3]{I_{3}}\right]$ over $\mathbb{B}^{\prime}=\operatorname{su}(2) \quad \mathfrak{s u}(3)$

All the formulae (4.2), (4.4) and (4.5) can be checked directly from the definitions concerned. A comparison of the commutative Galois extension structures on $\mathfrak{s u}(3)$ is visualized in Fig. 2. As we can see, $G_{1}$ is isomorphism of (sub)algebras induced by the fact that they are isomorphic to $\mathfrak{s u}(2)$ and $H_{1}$ is binary extension different for each (sub)algebra by the different choice of element $\tau$, that $\tau^{2}=I_{3}$.

\section{Identification of Construction of the Collection of Two Ternaries with the Collection of Three Binaries}

Starting with definitions [1]

$$
x_{1}+x_{2} i=x_{1}+x_{2} \sqrt{-1} \Leftrightarrow\left(\begin{array}{cc}
x_{1} & x_{2} \\
-x_{2} & x_{1}
\end{array}\right),
$$



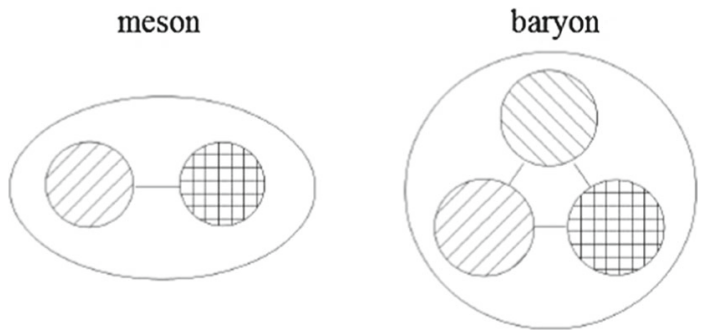

FiguRE 3. Examples of binary and ternary particles

$$
y_{1}+\mathbf{j} y_{2}+\mathbf{j}^{2} y_{3} \Leftrightarrow\left(\begin{array}{lll}
y_{1} & y_{2} & y_{3} \\
y_{3} & y_{1} & y_{2} \\
y_{2} & y_{3} & y_{1}
\end{array}\right) \quad\left(=Y_{1}\right)
$$

and

$$
Y_{2}=\left(\begin{array}{lll}
y_{4} & y_{5} & y_{6} \\
y_{6} & y_{4} & y_{5} \\
y_{5} & y_{6} & y_{4}
\end{array}\right) \quad\left(\Leftrightarrow y_{4}+\mathbf{j} y_{5}+\mathbf{j}^{2} y_{6}\right)
$$

with $x_{1}, x_{2}, y_{1}, \ldots, y_{6} \in \mathbb{R}$,

we observe that

$$
\left(\begin{array}{lll}
X_{1} & X_{2} & X_{3} \\
X_{3} & X_{1} & X_{2} \\
X_{2} & X_{3} & X_{1}
\end{array}\right) \Leftrightarrow\left(\begin{array}{cc}
Y_{1} & Y_{2} \\
-Y_{2} & Y_{1}
\end{array}\right)
$$

follows from the definitions of $X_{1}, X_{2}, X_{3}$ and $Y_{1}, Y_{2}$.

The identification includes a correspondence of the related ternary and binary Galois structures in the sense precised in assertions of Theorem 1-3 regarding the Galois extension structures on the nonion algebra (enlightening, in particular, the passage from cubic algebra $\mathbb{B}$ to the nonion algebra $\mathbb{N}$ ) and the analogous structure on $\mathrm{su}(3)$.

\section{An Analogue for Sects. 2-5: Two Ternaries vs. Three Binaries for Quarks and Elementary Particles}

At the beginning of this and next section we shortly present the idea which is essentially contained in $[11,27]$ and related papers. We present it here in a slightly altered form and order for the seek of completeness.

In the procedure (4.1)-(4.4) and Fig. 6 of [1] we may replace crystallographic lattices by quarks and elementary particles with the minimal requirements for their definition at the initial stage of model [7,8,11,19-21]. In particular, we may start with mesons and baryons as examples of binary and ternary particles (Fig. 3).

We know that each meson constitutes a quark and an anti-quark, and that each baryon constitutes only three quarks or anti-quarks. This generates the duality related with the ternary Pauli exclusion principles generalized by Kerner [11] and specifying in [27] by Th. 4 (Fig. 4). 

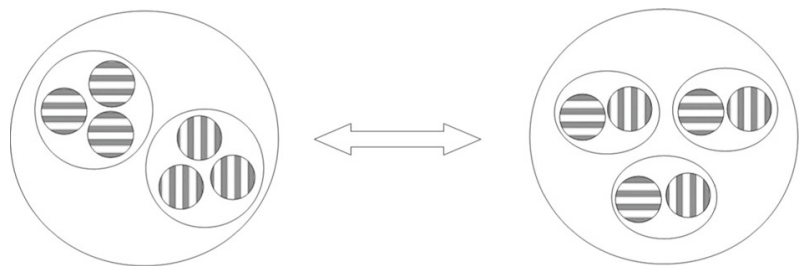

Figure 4. Duality related with the ternary Pauli exclusion principle

In turn we may study the quark realization of mesons and baryons by use of the binary Galois extension on $\mathfrak{s u}(3)$ [27], Figs. 3 and 4. We come to the quark realization of mesons and baryons by use of the binary resp. ternary Galois extension of $\mathfrak{s u}(3)$ refering to the Kobayashi-Masukawa theory; [27,28], Figs. 8, 9 and 10.

Following the Kobayashi-Masukawa model, we consider Galois extension of $\mathfrak{s u}(3): R[\sqrt{-1}, \sqrt{-1}, \sqrt[3]{1}, \sqrt{-1}]$. In this way we obtain six kinds of quarks. We remark that, usually, the Kobayashi-Masukawa theory is attributed to their study of two kinds of quarks.

Introducing the extension by $e_{0}(=\operatorname{diag}[1,1,0])$ we may identify the quarks as up-quark, down-quark, strange-quark, as follows

$$
\left\{e_{0}, e_{1}, e_{2}, e_{3}\right\} \Rightarrow u, \quad\left\{e_{0}^{\prime}, e_{1}^{\prime}, e_{2}^{\prime}, e_{3}^{\prime}\right\} \Rightarrow d, \quad\left\{e_{0}^{\prime \prime}, e_{1}^{\prime \prime}, e_{2}^{\prime \prime}, e_{3}^{\prime \prime}\right\} \Rightarrow s .
$$

Further, using the conjugate elements of (6.1)

$$
\left\{\bar{e}_{0}, \bar{e}_{1}, \bar{e}_{2}, e_{3}\right\} \Rightarrow \bar{u}, \quad\left\{\bar{e}_{0}^{\prime}, \bar{e}_{1}^{\prime}, \bar{e}_{2}^{\prime}, \bar{e}_{3}^{\prime}\right\} \Rightarrow \bar{d}, \quad\left\{\bar{e}_{0}^{\prime \prime}, \bar{e}_{1}^{\prime \prime}, \bar{e}_{2}^{\prime \prime}, \bar{e}_{3}^{\prime \prime}\right\} \Rightarrow \bar{s} .
$$

we realize mesons and baryons in Gell-Mann model by use of the Golois extension. Similarly, in the Kobayashi-Masukawa model-by use of the binary Galois extension structure of $\mathfrak{s u}(3)$ [27].

Noticing the duality for the 3-generation structures of quarks responsible for flavour; cf. [29]:

the binary structure of $\mathrm{u}, \mathrm{c}, \mathrm{b} \Longleftrightarrow$ the ternary structure of $\mathrm{u}, \mathrm{c}, \mathrm{b}$

the binary structure of $\mathrm{d}, \mathrm{s}, \mathrm{t} \Longleftrightarrow$ the ternary structure of $\mathrm{d}, \mathrm{s}, \mathrm{t}$ we arrive at a cumulative scheme for elementary particles including quarks, leptons and bozons, and generation of Matter (Fig. 5) as well as the corresponding 3-generations, more precisely: 3 objects consisting of 2 collections of 2 particles, constructed from 2 proper generations, more precisely from:

- 2 objects consisting of 3 collections of 2 particles (in each case),

- 2 objects consisting of 2 collections of 3 particles (in either case),

- 1 object consisting of 3 collections of 2 particles (in each case) and 1 object consisting of 2 collections of 3 particles (in either case).

It is natural to give an example of a corresponding 3-generation (Fig. 6). For introducing colours in terms of noncommutative Galois extensions we use the nonion extension of $\mathfrak{s u}(3)$; cf. the next section.

Summing up, we replace the programme $\boldsymbol{-}_{23}$ by the following 


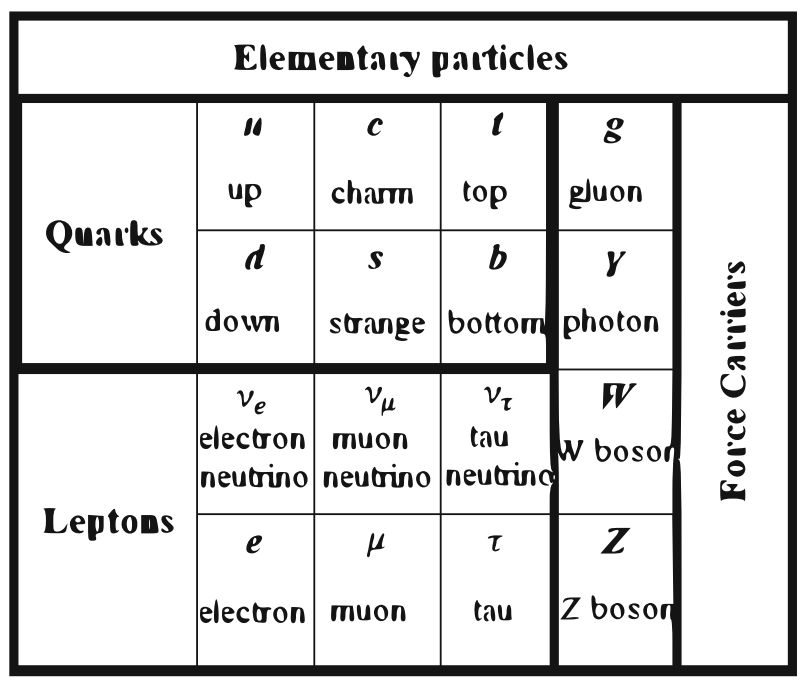

Figure 5. A cumulative scheme for elementary particles including quarks, leptons and bozons, and generations of matter

$\square_{13}$ binary and ternary elementary particles

$\square_{14}$ successive extensions of the related binary and ternary extensions on $\mathfrak{s u}(3)$

$\square_{15}$ relationships with the Gell-Mann model [13,14] and KobayashiMasukawa model [28]

$\square_{16}$ binary and ternary Pauli exclusion principles

$\square_{17}$ quark realization of mezons by the use of the binary Galois extension on $\mathfrak{s u}(3)$

$\square_{18}$ construction of quark models of mesons

$\square_{19}$ construction of quark models for baryons

$\square_{20}$ the 3-generations of quarks

$\square_{21}$ adjoint representations

$\square_{22}$ duality between the collections of corresponding binary and ternary Dirac operators

$\square_{23}$ generation of colours and the identification problem

The steps $\square_{13}-\square_{23}$ may be composed in the scheme shown on Fig. 7 . 

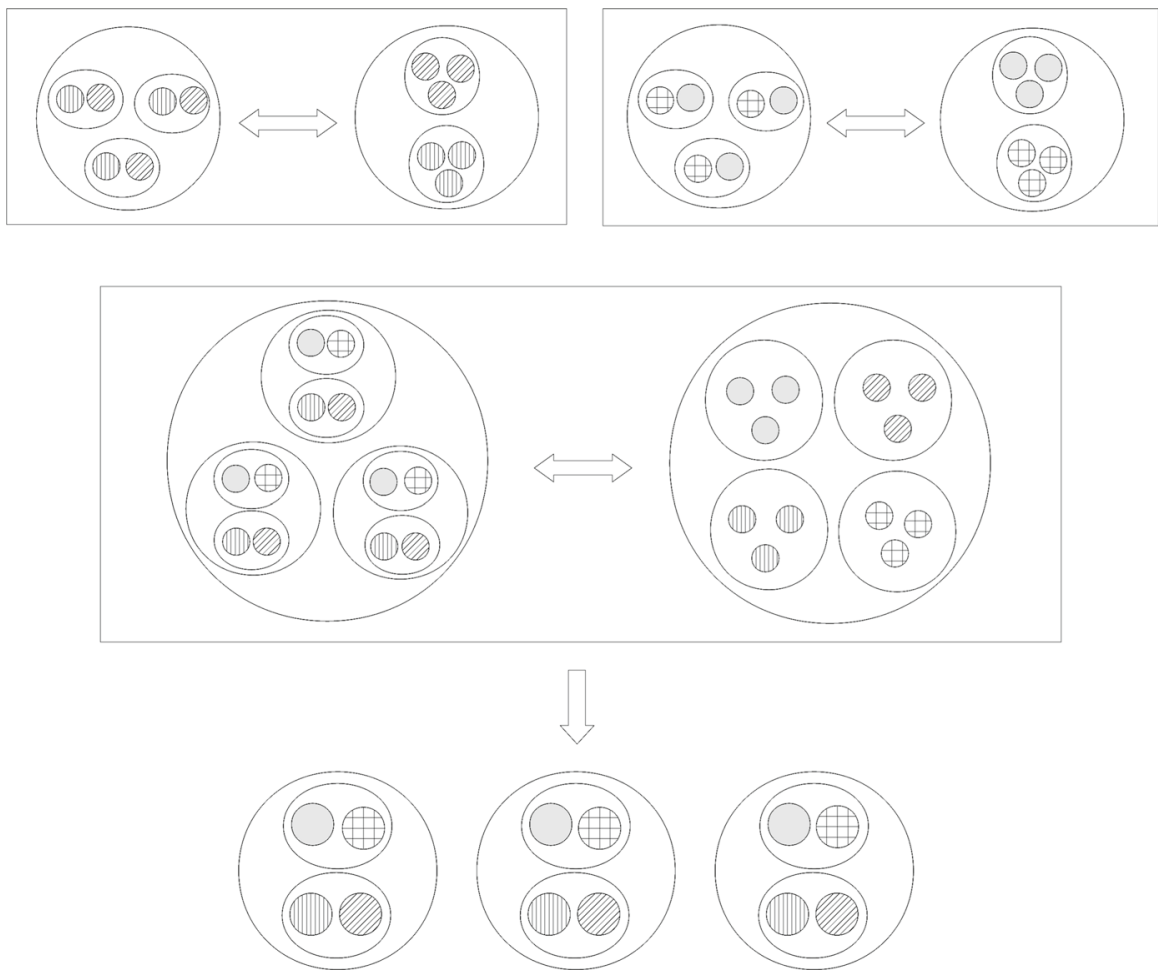

Figure 6. An example of a corresponding 3-generation constructed from 2 proper generations (proper in the sense described above)

\section{Introduction of Colours}

If we wish to introduce the concept of colours, we may consider the succesive ternary extension

$$
\mathbb{C}\left[\sqrt[2]{-I_{2}}, \sqrt[3]{I_{3}}, \sqrt[3]{I_{3}}\right] \simeq \operatorname{su}(3)\left[\sqrt[3]{I_{3}}\right]
$$

Indeed, $\mathbb{C}\left[\sqrt[2]{-I_{2}}\right]$ is the quaternionic algebra, so making its extension by $T_{2}$ as in (8) we get $\mathbb{C}\left[\sqrt[2]{-I_{2}}, \sqrt[3]{I_{3}}\right]$. Next, making the extension by the nonion algebra we arrive at $\mathbb{C}\left[\sqrt[2]{-I_{2}}, \sqrt[3]{I_{3}}, \sqrt[3]{I_{3}}\right]$, as desired. This means that we apply the nonion extension of $\mathfrak{s u}(3)$; cf. Sects. 1, 2, and 3:

$$
\tilde{\sigma}_{i} e_{k} \tilde{\sigma}_{i}^{-1}=e_{k}^{\prime \prime}, \quad i, k=1,2,3
$$

with $e_{k}, e_{k}^{\prime \prime}, k=1,2,3$, as in (17) and, in analogy to (21) and (22):

$$
\tilde{\sigma}_{1}=\left(\begin{array}{lll}
0 & 1 & 0 \\
0 & 0 & 1 \\
1 & 0 & 0
\end{array}\right), \quad \tilde{\sigma}_{2}=\left(\begin{array}{ccc}
0 & \mathbf{j}^{2} & 0 \\
0 & 0 & \mathbf{j} \\
1 & 0 & 0
\end{array}\right), \quad \tilde{\sigma}_{3}=\left(\begin{array}{ccc}
0 & \mathbf{j} & 0 \\
0 & 0 & \mathbf{j}^{2} \\
1 & 0 & 0
\end{array}\right), \quad \mathbf{j}^{3}=1, \quad \mathbf{j} \neq 1 .
$$




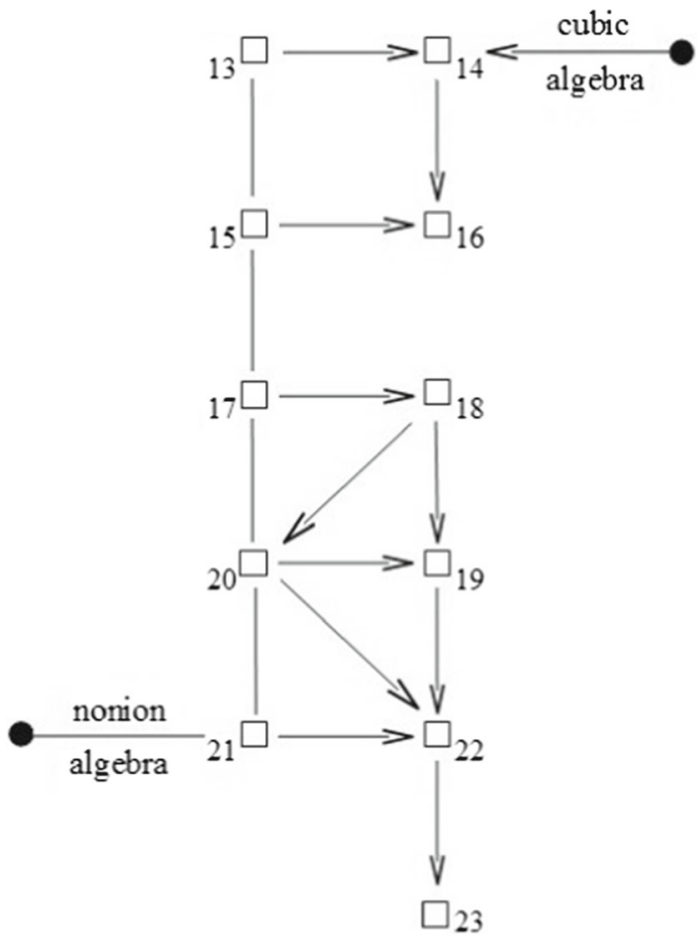

FiguRE 7 . Ternary approach related to the nonion algebra for quarks and elementary particles

For $\tilde{\sigma}_{1} e_{k} \tilde{\sigma}_{1}^{-1}$ we have $e_{k}$ and $e_{k}^{\prime \prime}, k=1,2,3$, as in (15) and

$$
\left\{\begin{array}{l}
\tilde{\sigma}_{1} e_{1} \tilde{\sigma}_{1}^{-1}=e_{1}^{\prime}, \quad \tilde{\sigma}_{1} e_{2} \tilde{\sigma}_{1}^{-1}=e_{1}^{\prime}, \quad \tilde{\sigma}_{1} e_{3} \tilde{\sigma}_{1}^{-1}=e_{3}^{\prime}, \\
\tilde{\sigma}_{1} e_{1}^{\prime} \tilde{\sigma}_{1}^{-1}=e_{1}^{\prime \prime}, \quad \tilde{\sigma}_{1} e_{2}^{\prime} \tilde{\sigma}_{1}^{-1}=e_{2}^{\prime \prime}, \quad \tilde{\sigma}_{1} e_{3}^{\prime} \tilde{\sigma}_{1}^{-1}=e_{3}^{\prime \prime}, \\
\tilde{\sigma}_{1} e_{1}^{\prime \prime} \tilde{\sigma}_{1}^{-1}=e_{1}, \quad \tilde{\sigma}_{1} e_{2}^{\prime \prime} \tilde{\sigma}_{1}^{-1}=-e_{2}, \quad \tilde{\sigma}_{1} e_{3}^{\prime \prime} \tilde{\sigma}_{1}^{-1}=e_{3} .
\end{array}\right.
$$

For $\tilde{\sigma}_{2} e_{k} \tilde{\sigma}_{2}^{-1}, k=1,2,3$, we have

$$
\begin{aligned}
& \tilde{e}_{1}=\left(\begin{array}{ccc}
0 & i & 0 \\
i & 0 & 0 \\
0 & 0 & 0
\end{array}\right), \quad \tilde{e}_{2}=i\left(\begin{array}{ccc}
0 & \mathbf{j} & 0 \\
\mathbf{j}^{2} & 0 & 0 \\
0 & 0 & 0
\end{array}\right), \quad \tilde{e}_{3}=\left(\begin{array}{ccc}
-i & 0 & 0 \\
0 & 0 & 0 \\
0 & 0 & i
\end{array}\right)=e_{3}^{\prime}, \\
& \tilde{\tilde{e}}_{1}=\left(\begin{array}{lll}
0 & 0 & \mathbf{j} \\
0 & 0 & 0 \\
\mathbf{j}^{2} & 0 & 0
\end{array}\right), \quad \tilde{e}_{2}=i\left(\begin{array}{ccc}
0 & 0 & \mathbf{j} \\
0 & 0 & 0 \\
\mathbf{j}^{2} & 0 & 0
\end{array}\right), \quad \tilde{\tilde{e}}_{3}=\left(\begin{array}{ccc}
0 & 0 & 0 \\
0 & 1 & 0 \\
0 & 0 & -1
\end{array}\right)=i e_{3}^{\prime \prime},
\end{aligned}
$$

and

$$
\left\{\begin{array}{l}
\tilde{\sigma}_{2} e_{1} \tilde{\sigma}_{2}^{-1}=\tilde{e}_{1}^{\prime}, \quad \tilde{\sigma}_{2} e_{2} \tilde{\sigma}_{2}^{-1}=\tilde{e}_{2}, \quad \tilde{\sigma}_{2} e_{3} \tilde{\sigma}_{2}^{-1}=\tilde{e}_{3}, \\
\tilde{\sigma}_{2} \tilde{e}_{1} \tilde{\sigma}_{2}^{-1}=\tilde{\tilde{e}}_{1}, \quad \tilde{\sigma}_{2} \tilde{e}_{2} \tilde{\sigma}_{2}^{-1}=\tilde{\tilde{e}}_{2}, \quad \tilde{\sigma}_{2} \tilde{e}_{3} \tilde{\sigma}_{2}^{-1}=\tilde{\tilde{e}}_{3}=-i e_{3}^{\prime \prime}, \\
\tilde{\sigma}_{2} \tilde{\tilde{e}}_{1} \tilde{\sigma}_{2}^{-1}=e_{1}, \quad \tilde{\sigma}_{2} \tilde{\tilde{e}}_{2} \tilde{\sigma}_{2}^{-1}=e_{2}, \quad \tilde{\sigma}_{2} \tilde{\tilde{e}}_{3} \tilde{\sigma}_{2}^{-1}=i e_{3} .
\end{array}\right.
$$



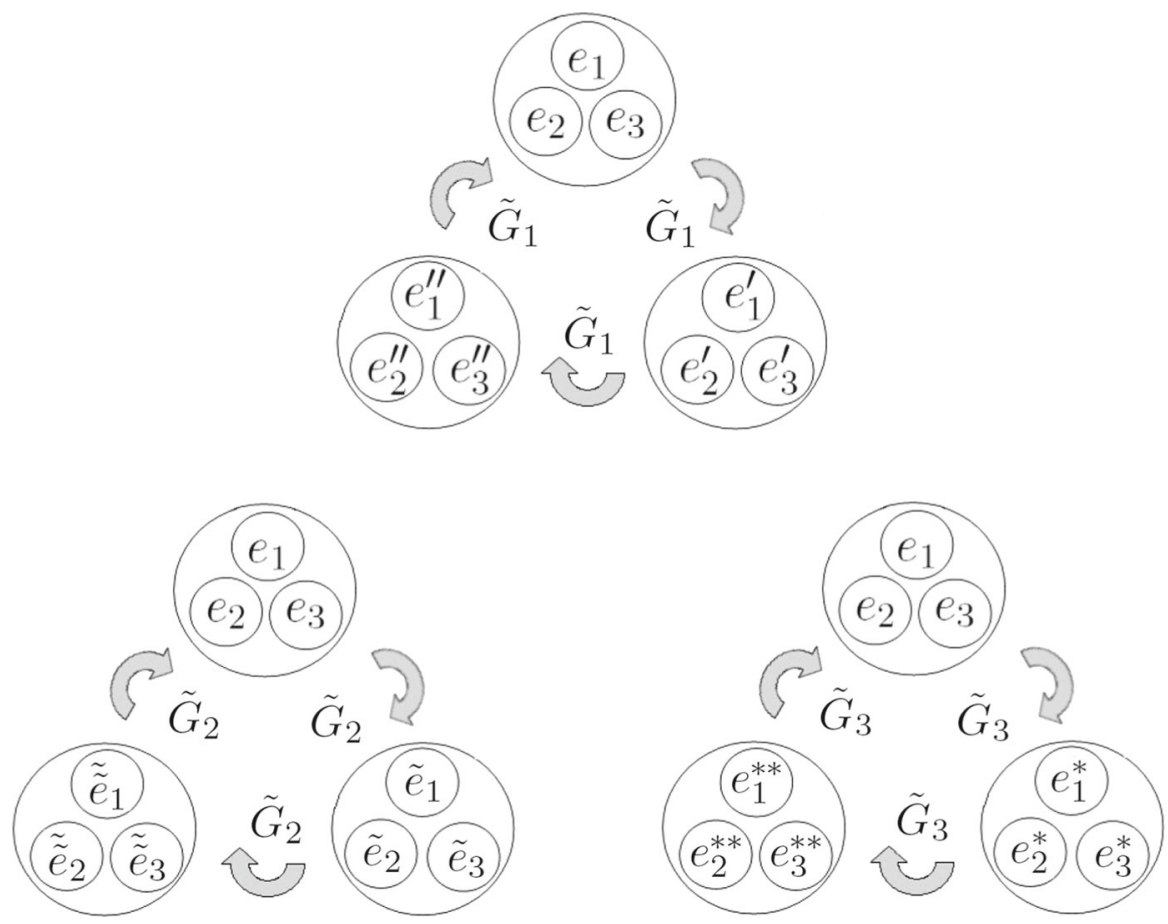

Figure 8. Generation of transformations $\tilde{\sigma}_{1}, \tilde{\sigma}_{2}, \tilde{\sigma}_{3}$ by the nonion extension of $\mathfrak{s u}(3)$

For $\tilde{\sigma}_{3} e_{k} \tilde{\sigma}_{3}^{-1}, k=1,2,3$, we have

$$
\begin{aligned}
& e_{1}^{*}=i\left(\begin{array}{ccc}
0 & 0 & \mathbf{j} \\
0 & 0 & 0 \\
\mathbf{j}^{2} & 0 & 0
\end{array}\right), e_{2}^{*}=i\left(\begin{array}{ccc}
0 & 0 & \mathbf{j} \\
0 & 0 & 0 \\
-\mathbf{j}^{2} & 0 & 0
\end{array}\right), e_{3}^{*}=\left(\begin{array}{ccc}
-i & 0 & 0 \\
0 & 0 & 0 \\
0 & 0 & i
\end{array}\right)=e_{3}^{\prime}, \\
& e_{1}^{* *}=i\left(\begin{array}{ccc}
0 & 0 & 0 \\
0 & 0 & \mathbf{j} \\
0 & \mathbf{j}^{2} & 0
\end{array}\right), e_{2}^{* *}=i\left(\begin{array}{ccc}
0 & 0 & \mathbf{j} \\
0 & 0 & 0 \\
\mathbf{j}^{2} & 0 & 0
\end{array}\right), e_{3}^{* *}=\left(\begin{array}{ccc}
i & 0 & 0 \\
0 & -i & 0 \\
0 & 0 & 0
\end{array}\right)=i e_{3},
\end{aligned}
$$

and

$$
\left\{\begin{array}{l}
\tilde{\sigma}_{3} e_{1} \tilde{\sigma}_{3}^{-1}=e_{1}^{*}, \quad \tilde{\sigma}_{3} e_{2} \tilde{\sigma}_{3}^{-1}=e_{2}^{*}, \quad \tilde{\sigma}_{3} e_{3} \tilde{\sigma}_{3}^{-1}=e_{3}^{*}, \\
\tilde{\sigma}_{3} e_{1}^{*} \tilde{\sigma}_{3}^{-1}=e_{1}^{* *}, \quad \tilde{\sigma}_{3} e_{2}^{*} \tilde{\sigma}_{3}^{-1}=e_{2}^{* *}, \quad \tilde{\sigma}_{3} e_{3}^{*} \tilde{\sigma}_{3}^{-1}=e_{3}^{* *}=e_{3}, \\
\tilde{\sigma}_{3} e_{1}^{* *} \tilde{\sigma}_{3}^{-1}=e_{1}, \quad \tilde{\sigma}_{3} e_{2}^{* *} \tilde{\sigma}_{3}^{-1}=e_{2}, \quad \tilde{\sigma}_{3} e_{3}^{* *} \tilde{\sigma}_{3}^{-1}=e_{3} .
\end{array}\right.
$$

the rules (7.1) or (7.2)-(7.4) for the transformations $\tilde{\sigma}_{1}, \tilde{\sigma}_{2}, \tilde{\sigma}_{3}$ are illustrated by the schemes in Fig. 8 .

The Dirac-like operators for

$$
\begin{aligned}
& \left(e_{0}^{\prime}, e_{1}^{\prime}, e_{2}^{\prime}, e_{3}^{\prime}\right), \quad\left(e_{0}^{\prime \prime}, e_{1}^{\prime \prime}, e_{2}^{\prime \prime}, e_{3}^{\prime \prime}\right), \quad\left(\tilde{e}_{0}, \tilde{e}_{1}, \tilde{e}_{2}, \tilde{e}_{3}\right), \\
& \left(\tilde{\tilde{e}}_{0}, \tilde{\tilde{e}}_{1}, \tilde{\tilde{e}}_{2}, \tilde{\tilde{e}}_{3}\right), \quad\left(e_{0}^{*}, e_{1}^{*}, e_{2}^{*}, e_{3}^{*}\right), \quad\left(e_{0}^{* *}, e_{1}^{* *}, e_{2}^{* *}, e_{3}^{* *}\right)
\end{aligned}
$$



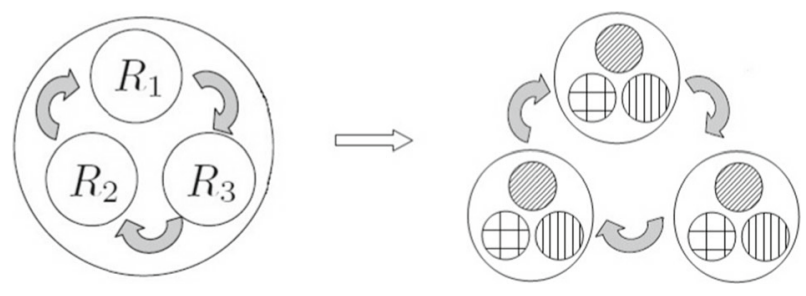

Figure 9. The concept of introducing colours for elementary particles via the transformations $\tilde{\sigma}_{1}, \tilde{\sigma}_{2}, \tilde{\sigma}_{3}$ governing the nonion extension of $\mathfrak{s u}(3)$

can be introduced in an analogous way as shown in Fig. 9. The proposed approach can be naturally modified for more general quark models what is planned for a future paper.

It is worth-while to notice several other important applications of Galois extensions and noncommutative Galois theory in contemporary physics [3037]. Also, because of an experimental confirmation of the existence of the Higgs boson [38-41], it is natural to extend our approach combining the Kobayashi-Masukawa model with the BEH-mechanism (here B stands for Robert Brout, E for Franois Englert, Nobel Prize 2013, and H for Peter W. Higgs, Nobel Prize 2013) [42-47]. In this context we can see an elegant interpretation of considering our approach parallelly in the theories of condensed matter and elementary particles - the appearance of the Nambu [48-50]-Goldstone [51] boson and the Meissner effect in superconductivity within the Ginzburg-Landau model: temperature-dependent vector potential and photons getting the mass. Around 1960 Yôichirô Nambu (Nobel Prize 2008) extended some ideas from superconductivity to particle physics. Jeffrey Goldstone introduced a complex massive scalar field and gave a theorem on appearance of a new massless scalar (Nambu-Goldstone) boson. Note that Murray Gell-Mann (Nobel Prize 1969) and Yuval Ne'eman in connection with the symmetry group $\mathrm{SU}(3)$ introduced the new quantum number called flavour [13].

\section{Conclusions}

For conclusions, we combine the step $\bullet_{6}$ of Sect. 2 in our previous paper [1] and the steps $\bullet \bullet_{13} \bullet_{23}$ formulated there in Sect. 6 with the steps $\boldsymbol{n}_{13} \boldsymbol{\square}_{23}$ :

binaries and ternaries; construction of the collection of two ternaries

ternaries and quaternaries; construction of the collection of three quaternaries 
the corresponding Dirac-like operators in connection with the

14 cubic algebra

the corresponding Dirac-like operators in connection with the nonion algebra

the Dirac-like operators corresponding to the collection of three

$\uparrow \bullet_{16}$ binaries in connection with the nonion algebra and its binary extension

$\downarrow \bullet 15$ construction of the collection of three binaries

$\boldsymbol{\square}_{15}$ construction of the collection four ternaries

Jordan-von Neumann-Wigner elements, complete elements, per-

6 fect elements; an example of perfect 15-element system

the corresponding Dirac-like operators in connection with the

$\boldsymbol{~}_{16}$ duodevicenion or quindenion algebra $[52,53]$ and its ternary extension

$\downarrow \bullet 16$ noncommutative Galois extensions for $\bullet_{16}$ and their basic relations

$\boldsymbol{\square}_{17}$ noncommutative Galois extensions for $\boldsymbol{\square}_{16}$ and their basic relations binary Galois extensions and Galois extensions of binary Clifford type for $\bullet 16$

ternary Galois extensions and Galois extensions of ternary Clifford type for $\boldsymbol{\nabla}_{16}$

ternary Galois extensions and Galois extensions of ternary Clifford

19 type for $\bullet 16$

quaternary Galois extensions and Galois extensions of quaternary Clifford type for $\boldsymbol{\bullet}_{16}$

Jordan-von Neumann-Wigner elements, complete elements, perfect elements; an example

the Galois extension structure of the duodevicenion and quindenion algebras

$\bullet_{20}$ the Galois extension structure of the nonion algebra

-21 ternary and binary Galois extension structure for $\mathfrak{s u}(3)$

$\mathbf{\square}_{21}$ quaternary and ternary Galois extension structure for $\mathfrak{s u}(3)$

binary and ternary Dirac-like operators of noncommutative Galois

- 22 extensions

ternary and quaternary Dirac-like operators of noncommutative Galois extensions identification of the constructed collection of two ternaries with

-23 the proper collection of three binaries

identification of the constructed collection of three quaternaries with the proper collections of four ternaries

The steps $\bullet_{6}, \bullet_{13}-\bullet_{23}, \boldsymbol{\bullet}_{13}-\boldsymbol{\bullet}_{23}$ may be composed in the scheme shown on Fig. 10 .

Other new ideas are provided by Perk [55] and Au-Yang and Perk [56], kindly communicated to us in a private letter of Prof. Perk $(21.08 ., 2014)$. 


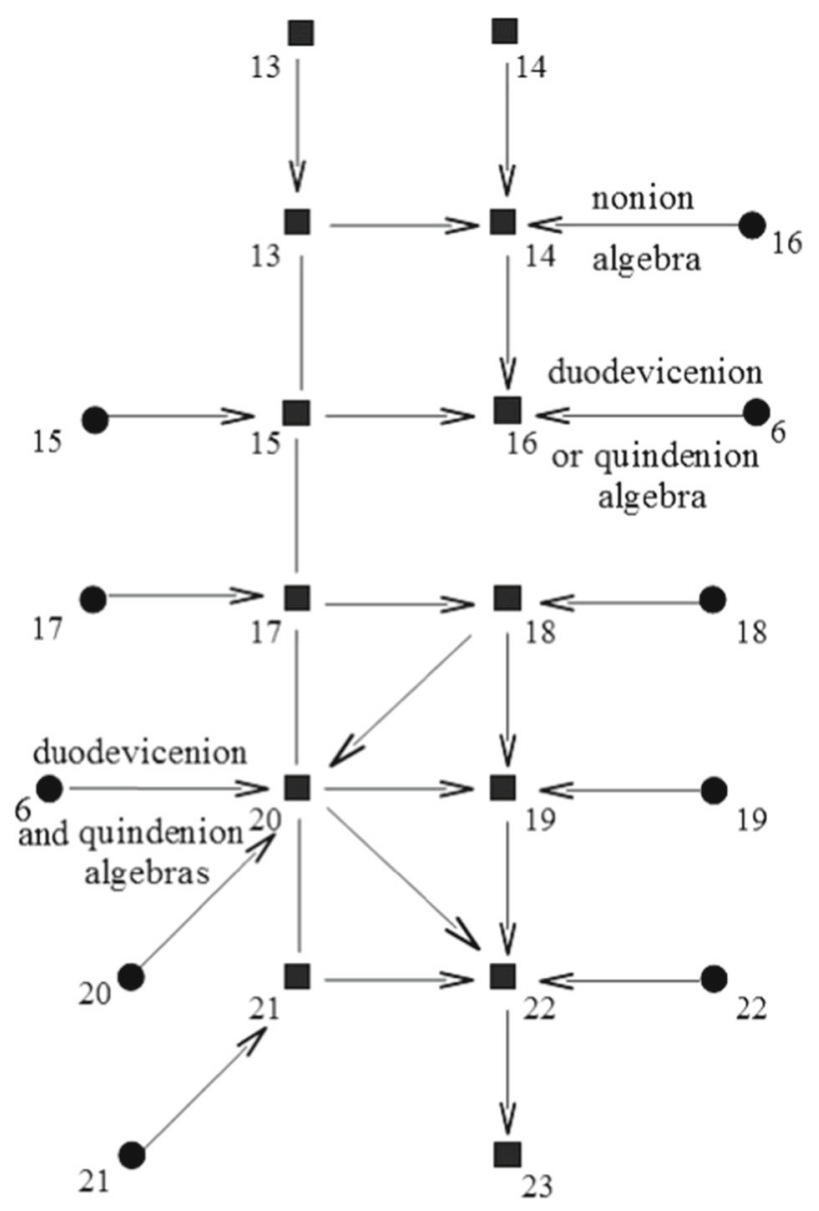

FiguRE 10. Quaternary approach related to the quindenion algebra, for crystallographic lattices

Open Access. This article is distributed under the terms of the Creative Commons Attribution 4.0 International License (http://creativecommons.org/licenses/by/4. 0/), which permits unrestricted use, distribution, and reproduction in any medium, provided you give appropriate credit to the original author(s) and the source, provide a link to the Creative Commons license, and indicate if changes were made.

Publisher's Note Springer Nature remains neutral with regard to jurisdictional claims in published maps and institutional affiliations.

\section{References}

[1] Ławrynowicz, J., Suzuki, O., Niemczynowicz, A., Nowak-Kȩpczyk, M.: Fractals and chaos related to Ising-Onsager lattices. Ternary approach versus binary 
approach. Int. J. Geom. Methods Mod. Phys. 15(11), 1850187 (2018). https:// doi.org/10.1142/S0219887818501876

[2] Ising, E.: Beitrag zur Theorie des Ferromagnetismus. Zschr. f. Phys. 31, 253$258(1925)$

[3] Onsager, L.: Crystal statistics. I. A two-dimensional model with an orderdisorder transition. Phys. Rev. 65, 117-149 (1944)

[4] Zhang, Z.-D.: Conjectures on exact solution of three-dimensional (3D) simple orthorhombic Ising lattices. Phil. Mag. 87, 5309-5419 (2007). [arXiv:0705.1045 [cond-mat] (pp.1-170)]

[5] Zhang, Z.-D.: Mathematical structure of the three-dimensional (3D) Ising model. Chinese Phys. B 22, 030513 (2013)

[6] Zhang, Z.-D., Suzuki, O., March, N.H.: Clifford algebra approach of 3D Ising model. Adv. Appl. Clifford Algebras 29, 12 (2019)

[7] Ławrynowicz, J., Nôno, K., Nagayama, D., and Suzuki, O., "Non-commutative Galois theory on Nonion algebra and $\mathrm{su}(3)$ and its application to construction of quark models", Proc. of the Annual Meeting of the Yukawa Inst. Kyoto "The Hierarchy Structure in Physics and Information Theory" Soryuusironnkennkyuu, Yukawa Institute, Kyoto, pp. 145-157 [http://www2. yukawa.kyoto-u.ac.jp] (2011)

[8] Ławrynowicz, J., Nôno, K., Nagayama, D., Suzuki, O.: A method of noncommutative Galois theory for binary and ternary Clifford Analysis. Proc. ICMPEA (Internat. Conf. on Math. Probl. in Eng. Aerospace, and Sciences) Wien, AIP (Amer. Inst. of Phys.) Conf. 1493, 1007-1014 (2012)

[9] Ławrynowicz, J., Nowak-Kȩpczyk, M., Suzuki, O.: Fractals and chaos related to Ising-Onsager-Zhang lattices vs. the Jordan-von Neumann-Wigner procedures. Quaternary approach. Internat. J. of Bifurcations and Chaos 22(1), 1230003 (19 pages) (2012)

[10] Ławrynowicz, J., Suzuki, O., Niemczynowicz, A.: Fractals and chaos related to Ising-Onsager-Zhang lattices vs. the Jordan-von Neumann-Wigner procedures. Ternary approach. Internat. J. of Nonlinear Sci. and Numer. Simul. 14(3-4), 211-215 (2013)

[11] Kerner, R.: $\mathbb{Z}_{3^{-}}$graded algebras and the cubic root of supersymmetry translations. J. Math. Phys. 33, 403-411 (1992)

[12] Kerner, R., Suzuki, O.: Internal symmetry groups of cubic algebra. Internat. J. of Geom. Methods in Modern. Phys. 9, 1261007 (10 pages) (2012)

[13] Gell-Mann, M., Ne'eman, Y.: The Eight-fold Way. W. A. Benjamin Inc, New York-Amsterdam (1964)

[14] Gell-Mann, M.: Symmetries of baryons and mesons. Phys. Rev. 125(3), 1067$1084(1962)$

[15] Ławrynowicz, J., Ne'eman, Y., Rembieliński, J., Szudy, J. and Wojtczak, L. eds.: Ideas of Albert Abraham Michelson in Mathematical Physics. I-II, Bull. Soc. Sci. Lettres Łódź 52-53 Sér. Rech. Déform. 38-39, (2002-2003), 167pp. + $165 \mathrm{pp}$

[16] Ławrynowicz, J., Marchiafava, S., Nowak-Kȩpczyk, M.: Periodicity theorem for structure fractals in quaternionic formulation. Internat. J. of Geom. Meth. in Modern Phys. 3, 1167-1197 (2006) 
[17] Ławrynowicz, J., Suzuki, O., Castillo Alvarado, F.L.: Basic properties and applications of graded fractal bundles related to Clifford structures. An introduction. Ukrain. Mat. Zh. 60, 603-618 (2008)

[18] Shaw, R.: Ternary composition algebras: 8 dimensions out of 4 ? Il nuovo Cimento 104 B(2), 161-183 (1989)

[19] Ławrynowicz, J., Nôno, K., Nagayama, D., Suzuki, O.: Binary and ternary Clifford analysis on nonion algebra and su(3). Bull. Soc. Sci. Lettres Łódź Sér. Rech. Déform 63(3), 33-48 (2013)

[20] Vaccaro, M.: Subspaces of a paraquaternionic Hermitian vector space. Internat. J. of Geom. Methods in Modern. Phys. 8(7), 1487-1506 (2011)

[21] Ławrynowicz, J., Vaccaro, M.: Structure fractals in para-quaternionic geometry. Ann. Univ. Mariae Curie-Skłodowska Sect. A Math. 65(2), 63-73 (2012)

[22] Kovacheva, R. K., Ławrynowicz, J., and Marchiafava, S. (eds.) Applied Complex and Quaternionic Approximation, Ediz. Nuova Cultura Univ. 'La Sapienza', Roma (2009) xxvi + 238pp

[23] Aubin, Th, Ławrynowicz, J., Wojtczak, L.: Nonlinear parabolic equations, relaxation and roughness. Bull. Soc. Sci. Math. (France) (2) 117, 313-327 (2003)

[24] Ławrynowicz, J., Polatoglou, H.M.: The relaxation and stochastical relaxation problems in crystals in terms of para-quaternions. Acta Physicae Superficierum 12, 97-107 (2012)

[25] Ławrynowicz, J., Marchiafava, S., Castillo Alvarado, F.L., Niemczynowicz, A.: (Para) quaternionic geometry, harmonic forms, and stochastical relaxation. Publ. Math. Debrecen 84(1-2), 205-220 (2014)

[26] Ławrynowicz, J., Nôno, K., Nagayama, D., Suzuki, O.: A method for noncommutative Galois theory and constructuion of quark models (KobayashiMasukawa model). I. Succesive Galois extensions. Bull. Soc. Sci. Lettres Łódź Sér. Rech. Déform. 63(1), 95-112 (2013)

[27] Ławrynowicz, J., Nôno, K., Nagayama, D., Suzuki, O.: A method for noncommutative Galois theory and construction of quark models (KobayashiMasukawa model). II. Exclusion principles, quark models and colours. Bull. Soc. Sci. Lettres Łódź Sér. Rech. Déform. 63(2), 79-95 (2013)

[28] Kobayashi, M., Masukawa, T.: CP-violation in the renormalizable theory of weak interaction. Progress Theor. Physics 49(2), 652-657 (1973)

[29] Huang, K.: Quarks, Leptons and Gauge Fields, 2nd edn. World Scientific, Singapore (2013)

[30] Brzeziński, T.: On modules associated to coalgebra Galois extensions. J. Algebra (215)(1), 290-317 (1999)

[31] Brzeziński, T., Hajac, P.M.: Coalgebra extensions and algebra coextensions of Galois type. Comm. Algebra 27(3), 1347-1367 (1999)

[32] Dąbrowski, L., Grosse, H., Hajac, P.M.: Strong connections and Chern-Connes pairing in the Hopf-Galois theory. Comm. Math. Phys. 220, 301-331 (2001)

[33] Brzeziński, T., Hajac, P.M.: The Chern-Galois character. C. R. Acad. Sci. Paris 338, 113-116 (2004)

[34] Krähmer, U., Zieliński, B.: On piecewise trivial Hopf-Galois extensions. Czech. J. Phys. 56(10/11), 1221-1226 (2006)

[35] Zieliński, B.: "Locally coalgebra-Galois extensions", [arXiv:math/0512150] 
[36] Hajac, P.M., Kröhmer, U., Matthes, R., Szymański, W., and Zieliński, B.: "Topological concepts in Hopf-Galois theory", In: Quantum Symmetry in Noncommutative Geometry, Hajac, P.M., ed., EMS Publ. House, to appear

[37] Hajac, P.M., Matthes, R., Sołtan, P.M., Szymański, W., Zieliński, B.: "HopfGalois extensions and $\mathrm{C}^{*}$-algebras," ibid., to appear

[38] Aaltonen, T., et al.: (CDF and Do Collaborations), "Higgs boson studies at the Tevatron". Phys. Rev. D 88, 052014 (2013). [arXiv:1303.6346]

[39] ATLAS Collaboration "Measurments of Higgs boson production and couplings in diboson final states with the ATLAS detector at the LHC", Phys. Lett. B 726, 88-89 (2013) [arXiv:1507.1427]

[40] CMS Collaboration "Study of the mass and spin-parity of the Higgs boson Candidate via its decays to Z boson pairs", Phys. Rev. Lett. 110, 081803 (2013); CMS Physics Analysis Summary, HIG-13-002-pas.pdf

[41] ATLAS Collaboration "Evidence for the spin-o nature of the Higgs boson using ATLAS data", Phys. Lett. B726, 120-121 (2013) [arXiv:1307.1432]

[42] The Royal Swedish Academy of Sciences "Class for Physics of the Royal Swedish Academy of Sciences, Scientific background on the Nobel Prize in Physics 2013: The BEH-mechanism interactions with short range forces and scalar particles", Stockholm, $28 \mathrm{pp}$

[43] Englert, F., Higgs, P.W.: Nobel Lecture 2013 in Physics. The Royal Swedish Academy of Sciences, Stockholm (2013)

[44] Higgs, P.W.: Broken symmetries, massless particles and gauge fields. Phys. Lett. 12, 132-133 (1964)

[45] Higgs, P.W.: Broken symmetries and the mass of the gauge bosons. Phys. Rev. Lett. 13, 508-509 (1964)

[46] Englert, F., Brout, R.: Broken symmetry and the mass of the gauge vector mesons. Phys. Rev. Lett. 13, 321-322 (1964)

[47] Higgs, P.W.: Spontaneous symmetry breakdown without massless boxons. Phys. Rev. 145, 1156-1166 (1966)

[48] Nambu, Y.: Quasi-particles and gauge invariance in the theory of super- conductivity. Phys. Rev. 117, 648-660 (1960)

[49] Nambu, Y.: "A superconductor' model of elementary particles and its consequencies", Talk given at a conference at Purdue (1960), reprinted In: Broken Symmetries. Selected Papers by Nambu, Y., ed. by Eguchi, T. and Nishijima, K., World Scientific, Singapore (1995)

[50] Han, M.Y., Nambu, Y.: Three-triplet model with double SU(3) symmetry. Phys. Rev. B 139, 1006-1020 (1965)

[51] Goldstone, J.: Field theories with superconductor solutions. Nuovo Cim. 19, 154-164 (1961)

[52] Nowak-Kępczyk, M.: An algebra governing reduction of quaternary structures to ternary structures I. Reductions of quaternary structures to ternary structures. Bull. Soc. Sci. Lettres Łódź Sér. Rech. Déform. 64((2)), 101-109 (2014)

[53] Nowak-Kȩpczyk, M. [2014] "An algebra governing reduction of quaternary structures to ternary structures II. A study of the multiplication table for the resulting algebra generators", Bull. Soc. Sci. Lettres Łódź Sér. Rech. Déform. 64(3), 81-90 (2014) 
[54] Nowak-Kȩpczyk, M.: An algebra governing reduction of quaternary structures to ternary structures III. A study of generations of the resalting algebras. Bull. Soc. Sci. Lettres Łódź Sér. Rech. Déform. 66(1), 81-90 (2016)

[55] Perk, J.H.H.: Comment on 'Mathematical structure of the three-dimensional (3D) Ising model. Chinese Phys. B 2+2(8), 080508 (5pp.) (2013). [arXiv:1307.1753v1]

[56] Au-Yang, H., Perk, J.H.H.: Parafermions of the tau-2 model. J. Phys. A: Math. Theor. 47, 315002 (19 pp.) (2014). [arXiv:1402.0061]

[57] Thouless, D.J., Duncan, F., Haldane, M., Kosterlitz, J.M.: Nobel Prize Lectures in Physics, Stockholm, (2016)

[58] Duncan, F., Haldane, M.: Nobel Lecture: Topological quantum matter. Rev. Mod. Phys. 89, 040502 (2017)

[59] Kosterlitz, J.M.: Nobel Lecture: Topological defects and phase transitions. Rev. Mod. Phys. bf 89, 040501 (2017)

[60] Duncan, F., Haldane, M.: Continuum dynamics of the 1-D Heisenberg antiferromagnet: Identification with the $\mathrm{O}(3)$ nonlinear sigma model. Phys. Lett. A 93, 464-468 (1983)

[61] Duncan, F., Haldane, M.: Nonlinear Field Theory of Large-Spin Heisenberg Antiferromagnets: Semiclassically Quantized Solitons of the One-Dimensional Easy-Axis Néel State. Phys. Rev. Lett. 50, 1153-1156 (1983)

[62] Duncan, F., Haldane, M.: Model for a Quantum Hall Effect without Landau Levels: Condensed-Matter Realization of the "Parity Anomaly". Phys. Rev. Lett. 61, 2015-2018 (1988)

[63] Kosterlitz, J.M.: The critical properties of the two-dimensional xy model. J. Phys. C: Solid State Phys. 7, 1046-1060 (1974)

[64] Kosterlitz, J.M., Thouless, D.J.: Ordering, metastability and phase transitions in two-dimensional systems. J. Phys. C: Solid State Phys. 6, 1181-1203 (1973)

[65] Kosterlitz, J.M., Thouless, D.J.: Long range order and metastability in two dimensional solids and superfluids. (Application of dislocation theory). J. Phys. C: Solid State Phys. 5, L124 (1972)

[66] Lipatov, L.N., Rausch de Traubenberg, M., Volkov, G.G.: On the ternary complex analysis and its applications. J. Math. Phys. 49, 013502 (2008)

[67] Trovon, A., Suzuki, O.: Noncommutative Galois Extensions and Ternary Clifford Analysis. Adv. Appl. Clifford Algebras 27, 59-70 (2017). https://doi.org/ 10.1007/s00006-015-0565-6

[68] Connes, A.: Noncommutative geometry. Academic, New York (1994)

[69] Ławrynowicz, J., Suzuki, O., Niemczynowicz, A., Nowak-Kȩpczyk, M.: "Fractals and chaos related to Ising-Onsager lattices. Relation to the Onsager model" In: Current Research in Mathematical and Computer Sciences II, ed. A. Lecko 131-140 (2018)

\section{J. Ławrynowicz}

Faculty of Physics and Applied Informatics

University of Łódź

Pomorska 149/153

90-236 Lodz

Poland

e-mail: jlawryno@uni.lodz.pl 
and

Institute of Mathematics

Polish Academy of Sciences

Śniadeckich 8

P.O.B. 2100-956 Warsaw

Poland

O. Suzuki

Department of Computer and System Analysis, College of Humanities and Sciences Nihon University

Sakurajosui 3-25-40

Setagaya 156-8550

Japan

e-mail: osuzuki1944butterfly@gmail.com

\author{
A. Niemczynowicz \\ Department of Analysis and Differential Equations \\ University of Warmia and Mazury \\ Słoneczna 54 \\ 10-710 Olsztyn \\ Poland \\ e-mail: niemaga@matman.uwm.edu.pl \\ M. Nowak-Kȩpczyk \\ Institute of Mathematics and Computer Science \\ The John Paul II Catholic University of Lublin \\ Al. Racławickie 14 \\ P.O. Box 12920-950 Lublin \\ Poland \\ e-mail: gosianmk@wp.pl
}

Received: September 24, 2018.

Accepted: March 19, 2019. 